\title{
Definition Class Scheme Item
}

National Cancer Institute

\section{Source}

National Cancer Institute. Definition Class Scheme Item. NCI Thesaurus. Code C42661.

A class that serves to allow many to many relationships between Definitions and ClassSchemeClassSchemeltem, providing uniqueness to the CS/CSI pairing to a definition. 